Title:

\title{
States in working memory store items with different memory quality
}

Author names and affiliations:

\section{Benjamin Peters ${ }^{1}$, Benjamin Rahm, ${ }^{2}$ Jochen Kaiser ${ }^{1}$, and Christoph Bledowski ${ }^{{ }^{*}}$}

${ }^{1}$ Institute of Medical Psychology, Goethe University, Frankfurt am Main, Germany

${ }^{2}$ Medical Psychology and Medical Sociology, Faculty of Medicine, University of Freiburg, Freiburg, Germany

* Corresponding author

Christoph Bledowski, PD Dr.

Institute of Medical Psychology

Goethe University

Heinrich-Hoffmann-Str. 10

60528 Frankfurt am Main Germany

Email: bledowski@em.uni-frankfurt.de 


\begin{abstract}
Working memory enables the storage of few items for a short period of time. Previous research has shown that items in working memory cannot be accessed equally well, indicating that they are held in at least two different states with different capacity limitations. However, it is unclear whether differences between states are due to limitations of the number of items that can be stored, or the quality with which items are stored. We employed a sequential whole-report procedure where participants reported the remembered orientation of each of 2 or 4 encoded Gabor patches. In addition, they rated their memory confidence prior to each report. Participants performed 600 trials per condition to obtain reliable subjective ratings and estimates of precision, guessing, and misreport using a mixture model, separately for each sequential report. Different measures of memory quality showed discontinuous trajectories consistently across reports with a steep drop from the first to the second remembered item but only slight decreases thereafter. In contrast, both reported and modeled guessing changed continuously across reports. Our results support the notion of two states in working memory and show that they are distinguished by memory quality rather than quantity. The first state stores high-quality representations that are highly susceptible to interference, whereas the second state contains lower-quality representations that are more robust against interference.
\end{abstract}




\section{Introduction}

Accessing relevant information even when it is no longer physically present is an essential component of goal-directed behavior. Working memory supports this ability by storing information over short periods of time, allowing its flexible access. However, the capacity of working memory is highly limited. Various accounts have been proposed to characterize the nature of this limitation. Some researchers have attributed it to a tightly limited number of discrete "slots" for storage (Zhang \& Luck, 2008), causing items to be forgotten when more items than slots are presented. Others have proposed that the capacity of working memory is best conceptualized as a continuous resource that is shared between all presented items (Bays \& Husain, 2008). Consequently, when more items are stored, less resource is available per item, resulting in a poorer memory quality.

Studies on capacity limitation typically assume that all items in working memory are held in the same single state. However, the general architecture of working memory is often conceived as comprising at least two different states of activation (Cowan, 1988; McElree, 2006; Oberauer, 2002; Unsworth \& Engle, 2007). One is a privileged state that keeps information active and readily available for cognition. The second state is commonly viewed as a passive state. Items require active retrieval to enter the privileged state of activation.

Support for this dual-state architecture of working memory came mainly from behavioral studies that have reported different reaction times for items that were designed to be held in different states (McElree, 2006; Oberauer, 2002) and by findings of separable brain regions involved in accessing items in different states (Bledowski, Rahm, \& Rowe, 2009; Nee \& Jonides, 2014). Recently, we have provided further evidence that items in working memory are stored in two qualitatively different states (Peters et al., 2018). In contrast to a typical working memory task where multiple items are encoded while only a single item is probed, we applied a procedure where sequentially all encoded items had to be reported. We estimated memory quality by calculating 'response precision' as the inverse width of the response error. In four experiments we consistently found that response precision decreased steeply after the first report but continued to decrease smoothly thereafter. This discontinuous trajectory of decrease in response precision across reports indicated that the first item was reported from a different state as compared to all subsequently reported items. Moreover, we also found that the first and the later reports were differentially sensitive to the experimental manipulation: Only the precision of the first report was reduced by interference during the retention period. Recently, Adam et al. (Adam, Vogel, \& Awh, 2017) also applied a sequential whole-report procedure to assess memory precision as a function of report order. When the sequence in which items had to be reported was randomized as in our study, they also found that memory precision dropped discontinuously across reports. Together, consistent findings across multiple experiments have indicated that prior to the first report, or prior to interference, items were held in a state of high resolution. Thereafter, items fell into a second state of lower resolution.

The notion of two states in working memory that store items with different quality seems a plausible interpretation for these findings. It is, however, possible that the observed decline in precision for the later reports was not due to a limited storage quality but to a limitation of the number 
of items that can be stored in the second state. Specifically, after the first report, or after interference, only a subset of the presented items may be stored in the second state. Hence, forcing participants to report all the presented items could lead to an increased guessing rate, resulting in an overall precision decline across trials for reports from the second state.

Mixture models are commonly applied to disentangle different sources that contribute to response precision. In such a model, the response error distribution can be reproduced by several components. The first component is a Gaussian distribution centered on the correct feature value of the reported item. The concentration of this distribution (model parameter $\mathrm{k}$ ) reflects the memory quality of the memory representation of this item. While this component is often called 'precision', here we use the term 'modeled precision' to avoid confusion with the above mentioned 'response precision' that is calculated as the inverse width (s.d.) of the response error distribution. A second component refers to a uniform distribution that is meant to correspond to the proportion of trials on which participants respond at random (modeled guessing). Bays et al. (2009) have proposed a third component that reflects responses based on erroneous recall of nontarget features, i.e., features of an item that formed part of the memory display but was not the one probed for recall (modeled misreport). While conceptually compelling and widely used, mixture modeling has recently been criticized. First, a valid separation of modeled precision and guessing might not be possible in conditions where participants perform poorly (Lawrence, 2010). Second, response errors may not represent a linear measure. Schurgin, Wixted, \& Brady (2018) have suggested that they are rather scaled to reflect 'psychological distance'. Consequently, error responses with a high deviation from the true value were suggested not to reflect guessing but are conceived as memories with low quality. However, another way to dissociate low-quality memory from guessing is to obtain memory confidence ratings for each report. Subjective ratings correlate with response precision, modeled precision and guessing, indicating that people have reasonable metacognitive knowledge about their memory (Adam et al., 2017; Rademaker, Tredway, \& Tong, 2012; van den Berg, van den Berg, \& Ma, 2017). Confidence ratings and reported feature values thus provide separate estimates of both memory quality and guessing across the report sequences.

To test whether the response precision difference between the two states in working memory was attributable to memory quality or to the number of items that can be stored, the present study used both objective and subjective measures as well as their combination. Specifically, as in our previous study we employed the whole-report procedure by asking participants to successively report each of 2 or 4 encoded Gabor patches from working memory. In addition, we also incorporated subjective confidence ratings of memory quality just before each report of the whole-report procedure. Participants performed 600 trials per condition on four days to obtain reliable estimates of subjective ratings of memory quality as well as modeled precision, guessing, and misreport using the mixture model, separately for each sequential report. We hypothesized that if the two states in working memory are distinguished by memory quality, this should be reflected by a difference in modeled precision and in the proportion of high confidence ratings. In contrast, if the second state is characterized by a limitation of the number of stored items, both states should differ mainly in the level of self-reported and modeled guessing and/or the number of modeled misreports. 


\section{Methods}

\section{Participants}

Ten students (5 women; average age 21.7 years) of Goethe University Frankfurt or Fresenius University of Applied Sciences Frankfurt with normal or corrected-to-normal vision participated in the study. Participants received financial compensation ( $€ 80$ for approximately $8 \mathrm{~h}$ ) and gave written informed consent. This research was approved by the ethics committee of the Goethe University Medical Faculty.

\section{Procedure}

The experimental paradigm including the whole-report procedure was comparable to our previous study (Peters et al., 2018). At the beginning of each trial a memory array of two or four randomly oriented Gabor patches was presented for $400 \mathrm{~ms}$ (Figure 1a). Their orientations differed by at least $5^{\circ}$ between stimuli. Gabor patches had a Gaussian envelope of $0.8^{\circ}$ and a wavelength of 0.8 cycles per degree. They could appear at eight possible locations equidistantly placed on an invisible circle with a radius of $8.2^{\circ}$ around the center of the screen. After a retention interval of $1,000 \mathrm{~ms}$, a white circle with a radius of $2^{\circ}$ centered on the location of one of the items was highlighted. Participants were asked first to report their confidence about their memory at that location on a 4-point scale from 0 (forgotten, i.e. no memory at all) to 3 (best possible memory) by keyboard button press. Then, a randomly oriented Gabor appeared at that location and participants adjusted the orientation of the probe to match the remembered orientation as closely as possible by horizontal mouse movements. The final adjustment was confirmed with a mouse click. This procedure was repeated until all memorized items, i.e., two or four depending on the set size, were reported. The sequence of reports was randomized and every item was reported only once. No response time limit was imposed. After the last report participants received feedback concurrently for all reports, in the form of small colored discs presented centrally on an invisible circle with a radius of $0.8^{\circ}$ around the fixation cross $\left(0.66^{\circ}\right)$ at locations that corresponded clearly to locations of probed orientations in the current trial. Colors of the discs ranged from green to red, with green indicating perfect report and red meaning that the adjusted probe orientation was perpendicular to the presented Gabor. Feedback was presented for $500 \mathrm{~ms}$. Trials were separated by a blank inter-trial interval of $1,000 \mathrm{~ms}$. Participants were encouraged to move their eyes during the inter-trial interval only.

The experiment was programmed in Presentation (Version 14.9, Neurobehavioral Systems, Inc., Berkeley, CA) and presented via an LCD monitor with a $60-\mathrm{Hz}$ refresh rate. Participants conducted 30 practice trials and subsequently completed 10 blocks of 30 trials in each of four sessions on separate days. This resulted in 600 trials per set size.

\section{Data analysis}

For each trial, we computed response errors as the angular difference between the presented orientation and the response given by the participant. To compare the results with our previous study (Peters et al., 2018), we first computed response precision as the inverse of the SD of the response error distribution, corrected for the expectation value under uniform response errors 
(Bays, Catalao, \& Husain, 2009). Response precision was calculated separately for each participant and condition. Moreover, we computed precision for each of four levels of reported confidence.

Confidence ratings were analyzed in two ways. In a first analysis, we averaged the confidence ratings for each report position, set size, and participant. Subsequently, we analyzed separately the proportion of high confidence ratings (rating $=3$ ) and the proportion of ratings that indicated forgetting (rating $=0$ ).

To assess the impact of sequential report position, we conducted two repeated-measures ANOVAs with report position as a single factor, separately for set size two and set size four, with reports 1-2 and reports 1-4 as levels, respectively. In addition, to assess the impact of set size, and its interaction with report position, we computed a $2 \times 2$ repeated-measures ANOVA that included the factor report position with the first two reports as its levels and the factor set size with the levels two and four. Whenever assumptions of sphericity were violated as indicated by a significant Mauchly test statistic $(p<.05)$, Greenhouse-Geisser-corrected degrees of freedom are reported.

\section{Mixture modeling}

To quantify the contribution of different sources of error to overall response precision estimates, we modeled the response error distributions with a mixture model comprising three parameters (Bays et al., 2009): 1) Reporting the cued item with probability ptarget from a circular normal distribution centered on the original orientation and with precision $\mathrm{K} . \mathrm{K}$ thereby directly reflects the memory quality with which an item is available for report (modeled precision), 2) guessing with probability pguessing in case that the target item cannot be retrieved (modeled guessing), or 3 ) incorrectly reporting another, uncued item with probability pnonTarget (modeled misreport). In the case of guessing, the response error is sampled from a uniform distribution on the support of $-\pi$ to $\pi$. Estimates of ptarget, PnonTarget and $\mathrm{k}$ were obtained as those parameters that minimized the negative log-likelihood of the response errors under the model. In order to enforce the constraint that $p_{\text {target }}+p_{\text {nonTarget }}+p_{\text {guessing }}=1$, we performed parameter optimization on transformed parameters pmemory, as $p_{\text {target }}+p_{\text {nonTarget, }}$ and $p_{\text {targets OfMemory }}$ as $p_{\text {target }} /\left(p_{\text {target }}+p_{\text {nonTarget }}\right)$. After optimization,

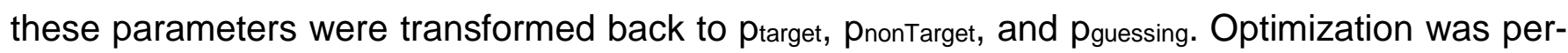
formed with the Nelder-Mead method as implemented in the function fminsearch in MATLAB. We used fminsearchbnd to constrain parameters between 0 as a lower limit and 1, 1, and 100 for pmemory, PtargetsofMemory and $\mathrm{k}$ as upper limits, respectively. Parameters were estimated independently for each set size, report position, and participant.

\section{Continuous vs. discontinuous model comparison}

To replicate the findings of our previous study, we tested whether the trajectory of a certain parameter value (i.e., response precision, mean rating, modeled precision, modeled guessing, modeled misreport, proportion of "high confidence" ratings and "forgotten" ratings) across the report sequence was more compatible with a one-state model or a two-state model by performing a formal model comparison between a continuous model and a discontinuous model. Specifically, reports from a single state should entail a constant rate of degradation across reports resulting in 
an exponential (continuous) decrease of this parameter across the report sequence. The parameter value $\mathrm{y}$ of report at position $\mathrm{x}$ by participant $\mathrm{i}$ was modeled as

$$
y_{i}(x)=a_{i} \exp \left(-b_{i}(x-1)\right)+\epsilon_{\mathrm{ix}}
$$

where $b_{i} \sim \mathcal{N}\left(b, \sigma_{b}\right)$ (slope parameter) and $a_{i} \sim \mathcal{N}\left(a, \sigma_{a}\right)$ (initial parameter value at the first report) were random effects parameters and $\varepsilon_{i x} \sim \mathcal{N}(0, \sigma)$ the error term. Positive values of the slope parameter $b$ indicated a gradual decline of the parameter across the report sequence. For the twostate model, we fit a discontinuous model that allowed for a discontinuity of the parameter trajectory after the first report. Specifically, we modified the exponential model to allow for a deviation of the first report position from the exponential trajectory at the first report position. Here, the parameter value $y$ of participant $\mathrm{i}$ at report position $\mathrm{x}$ was modeled as

$$
y_{i}(x)=a_{i} \exp \left(-b_{i}(x-1)\right)+c_{i} 1_{\{1\}}(x)+\epsilon_{i x},
$$

with $\mathbf{1}_{\{1\}}(x)$ being the indicator function (hence $\mathbf{1}_{\{1\}}(1)=1$ and $\mathbf{1}_{\{1\}}(x)=0$ for all $x \neq 1$ ). The random effects parameter $c_{i} \sim \mathcal{N}\left(b, \sigma_{c}\right)$, therefore captured the deviation of an exponential fit from the parameter value measured at the first report position.

To test whether response precision, mean rating, modeled precision, modeled guessing, modeled misreport, proportion of "high confidence" ratings and "forgotten" ratings changed continuously (or discontinuously), we fitted the continuous and discontinuous model to these parameters as described above. However, for the model comparison with modeled guessing, modeled misreport, and proportion of "forgotten" ratings we first subtracted these parameters from 1. Correspondingly, the c-parameters were then sign-flipped afterwards. This is because the successive deterioration across reports is reflected by an increase in these parameters, while the exponential term is only able to capture a degradation across reports.

We compared the fit of the exponential model with the fit of the discontinuous model using respective (marginal) Akaike Information Criteria (AIC). Because AIC is a relative fit index, we reported the difference $\triangle \mathrm{AIC}$ as the AIC of the discontinuous model minus the AIC of the exponential model. Thus, negative AIC values denoted evidence for the discontinuous model. Furthermore, because the exponential model was nested within the discontinuous model, the likelihood ratio of the two models was asymptotically $x^{2}(d f)$-distributed, with $d f$ as the number of constrained parameters as degrees of freedom. We therefore tested whether relaxing model constraints by introducing a new parameter (first report benefit parameter for the discontinuous model) significantly increased the model likelihood by computing a likelihood ratio test.

\section{Results}

\section{Response precision decreases discontinuously across the report sequence}

The current results replicated the findings from our previous study (Peters et al., 2018) by showing a discontinuous decrease of response precision, measured as the width of the response error distribution (inverted s.d.). That is, we observed a steep drop from the first to the second report and only a slight continuous decrease thereafter (Figure 1b, 1c). Response precision significantly decreased across the report sequence in both set size conditions (main effect of report position 


\section{Figure 1}

a

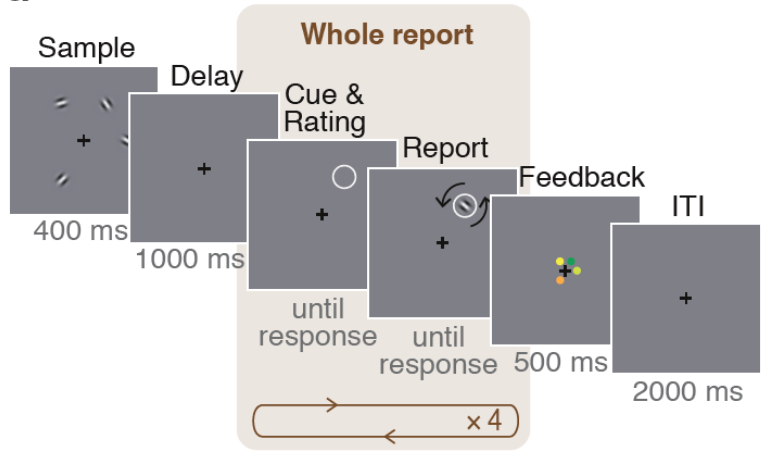

b

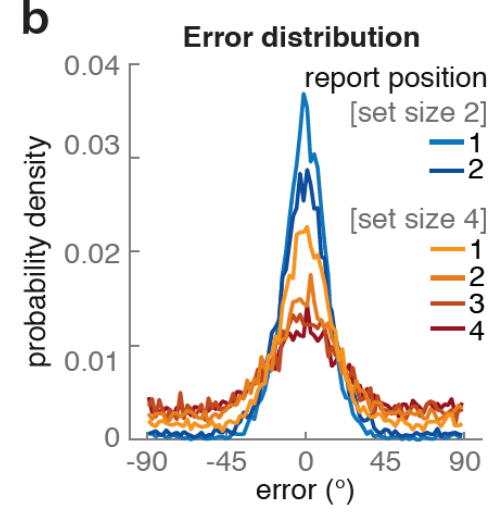

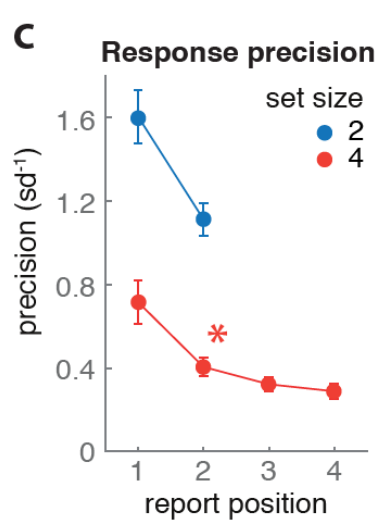

Figure 1. Paradigm and results of error distribution and response precision. a) Participants memorized two or four orientations of Gabor gratings and after a delay were asked to rate their confidence about the memory quality and to report the memorized orientation for each of them in random order (only set size 4 displayed). b) Distribution of response errors for each set size and report position and c) the corresponding response precision. Error bars indicate standard error of the mean across participants. Asterisk denotes an advantage of a discontinuous over a continuous model for the decline trajectory across reports.

for set size two: $F(1,9)=39.5, p=.0001, \eta_{p}^{2}=.81$; for set size four: $F(1.1,9.5)=26.5, p<.0001$, $\left.\eta_{p}^{2}=.74\right)$. Importantly, the decrease in response precision was better explained by the discontinuous model than by the exponential model $\left(\triangle \mathrm{AIC}=-34.43, \mathrm{X}^{2}(2)=38.43, p<.001, \mathrm{C}=0.245\right)$, confirming a discontinuity in the trajectory of response precision across report positions.

\section{Confidence ratings predict response precision}

To test whether confidence ratings represent a viable measure of memory quality, we compared statistically the width of the response error distribution between confidence ratings (Figure 2a). Response precision was significantly modulated by confidence ratings (main effect of confidence ratings: $\left.F(1.5,13.1)=129.7, p<.0001, \eta_{p}^{2}=.935\right)$. Response precision was highest when participants rated their memory quality to be highest and close to zero when they stated they had forgotten the item (mean response precision for rating 3: $1.32, \pm 0.09$, mean \pm standard error, mean response precision for rating $2: 0.66, \pm 0.06$ mean response precision for rating $1: 0.26, \pm 0.05$, mean response precision for rating $0: 0.09, \pm 0.02$ ). This confirms that confidence ratings provide a valid measure of memory quality (Rademaker et al., 2012).

\section{Confidence decreases discontinuously across the report sequence}

In the next step, we evaluated whether the confidence ratings across successive reports also revealed a discontinuous decline as observed for response precision. We therefore averaged the confidence ratings for each report position and set-size to obtain an estimate of the mean confidence. Figure $2 \mathrm{~b}$ shows that mean confidence ratings across reports followed a similar trajectory as response precision. Mean confidence dropped steeply from the first to the second report, but decreased only slightly thereafter in the set size four condition (main effect of report position for set size four: $F(3,27)=42.3, p<.0001, \eta_{p}^{2}=.82$ ). For set size two, the main effect of report position was not significant $\left(F(1,9)=3.8, p=.0834, \eta_{p}^{2}=.30\right)$. Importantly, as for response precision, the model comparison for confidence in the set size four condition clearly favored the discontinuous 

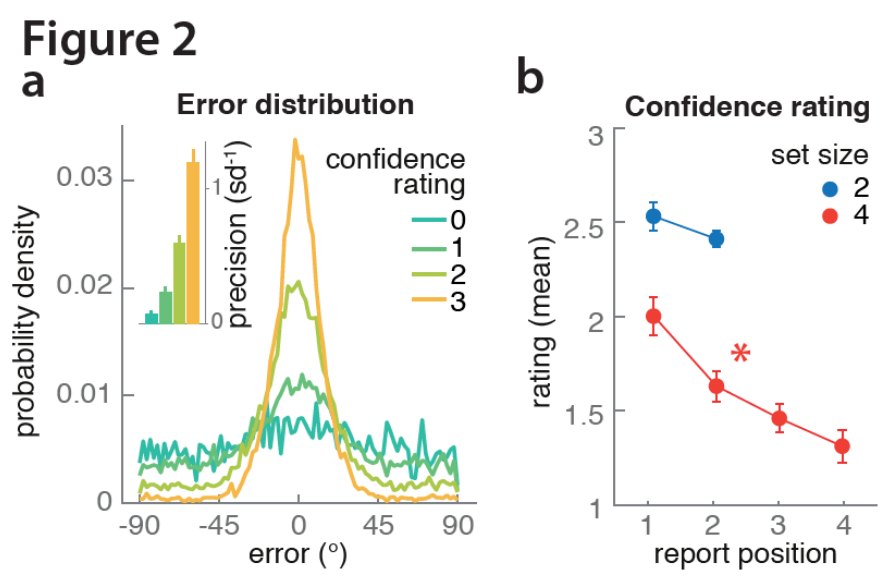

Figure 2. Confidence rating results. a) Distribution of response errors associated with each rating collapsed across set sizes. Inset barplot denotes the corresponding response precision. b) Mean rating for each report position and set size. Error bars indicate standard error of the mean across participants. Asterisk denotes an advantage of a discontinuous over a continuous model for the decline trajectory across reports.

over the continuous model $\left(\triangle \mathrm{AIC}=-5.14, \mathrm{X}^{2}(2)=9.14, p=.010, \mathrm{c}=0.248\right)$. This result confirmed a discontinuity in the trajectory of subjective mean confidence ratings across report positions. Again, these results support previous findings (Rademaker et al., 2012) that confidence ratings provide a valid measure of the quality of a memory representation.

\section{Modeled precision is responsible for the discontinuous decrease in response precision}

To investigate the possible sources producing the discontinuous trajectory of response precision decrease across reports, we modeled the response error distributions with a mixture model that assumes three potential sources of errors: modeled precision, guessing and misreport. As shown in Figure $3 \mathrm{~b}$, modeled precision decreased across the report sequence in both set size conditions (main effect of report position for set size two: $F(1,9)=26.0, p=.0006, \eta_{p}^{2}=.74$; for set size four: $\left.F(1.6,14.7)=7.7, p=0.007, \eta_{p}^{2}=.46\right)$. Similarly, we observed a significant change in guessing across reports in both set size conditions (main effect of report position for set size two: $F(1,9)=$ 16.8, $p=.0027, \eta_{p}^{2}=.65$; for set size four: $\left.F(3,27)=16.6, p<.0001, \eta_{p}^{2}=.65\right)$. In contrast, misreport was unaffected by report position in either condition (main effect of report position for set size two: $F(1,9)=0.5, p=.5108, \eta_{p}^{2}=.05$; for set size four: $F(3,27)=0.7, p=.5519, \eta_{p}^{2}=.07$ ).

Most importantly, we found that only modeled precision varied discontinuously between report positions. It dropped markedly from the first to the second report but decreased only very slightly thereafter (set size four condition). This discontinuous trajectory was supported by the model comparison that favored the discontinuous over the continuous model $\left(\triangle \mathrm{AIC}=-7.95, \mathrm{x}^{2}(2)=\right.$ $11.95, p=.0025, \mathrm{C}=1.857$ ). In contrast, we found a continuous rather than discontinuous trajectory for both other parameters. For guessing, the additional parameter of the discontinuous model did not yield any significant improvement of the data likelihood over the continuous model $\left(\mathrm{X}^{2}(2)\right.$ $=0.28, p=.870, \mathrm{c}=-0.016)$. Model comparison favored the continuous model $(\triangle \mathrm{AIC}=3.72)$ confirming the absence of a discontinuity after the first report position. Similarly, misreport was also better captured by a continuous model as the data likelihood was not significantly increased by the discontinuous model $\left(\triangle \mathrm{AIC}=-0.18, \mathrm{X}^{2}(2)=4.18, p=.124, \mathrm{C}=-0.117\right)$. 


\section{Figure 3}

a

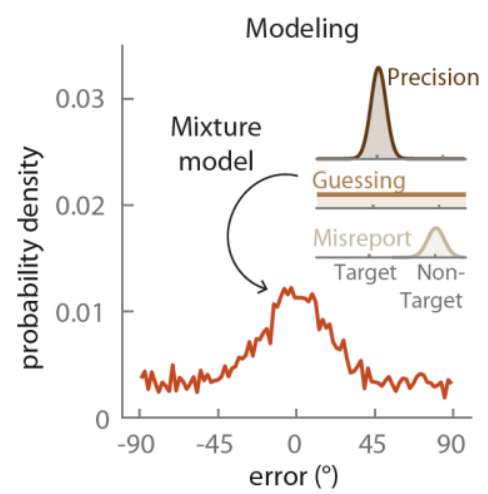

b

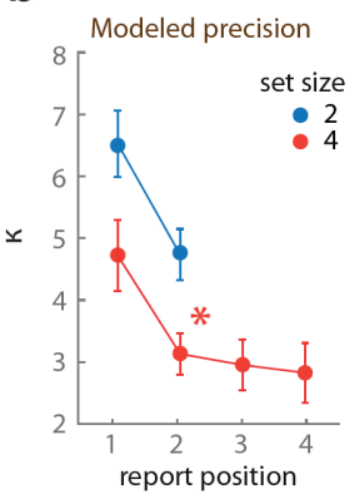

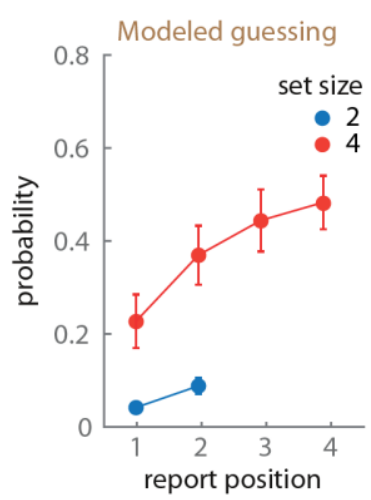

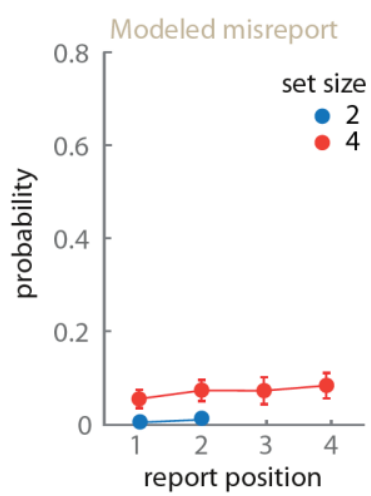

Figure 3. Mixture modeling. a) The mixture model estimates three parameters that reproduce the distribution of response errors: precision ( $\mathrm{k}$ : inverse variance of target-centered Gaussian), guessing and misreports. b) Maximum likelihood estimates of the three mixture model components. Error bars indicate standard error of the mean across participants. Asterisk denotes an advantage of a discontinuous over a continuous model for the decline trajectory across reports.

For the sake of completeness, we also report the effect of set size and its interaction with report position on response precision as well as on the three model parameters as indicated by the corresponding $2 \times 2$ ANOVA with the factors set size (2 and 4) and report position (1 and 2). Consistent with a large body of working memory literature (Bays et al., 2009; Wilken \& Ma, 2004; Zhang \& Luck, 2008), we found a significant decrease in response precision from set size two to set size four $\left(F(1,9)=107.4, p<.0001, \eta_{p}^{2}=.92\right)$. The set size effect was also clearly present for all three model parameters (modeled precision loss: $F(1,9)=62.6, p<.0001, \eta_{p}^{2}=.87$; guessing: $F(1,9)=22.0, p=.0011, \eta_{p}^{2}=.71$; and misreport: $\left.F(1,9)=9.0, p=.0150, \eta_{p}^{2}=.50\right)$. We also observed that the drop of response precision and the increase of guessing from the first to the second report was steeper for set size two than set size four. This was reflected by a significant interaction between report position and set size (response precision: $F(1,9)=5.5, p=.0441, \eta_{p}^{2}=$ .38; guessing: $\left.F(1,9)=6.2, p=.0344, \eta_{p}^{2}=.41\right)$. There were no such interactions for modeled precision $\left(F(1,9)=0.2, p=.6584, \eta_{p}^{2}=.02\right)$ or misreport $\left(F(1,9)=1.0, p=.3444, \eta_{p}^{2}=.10\right)$.

\section{Response precision decreases discontinuously even for high confidence ratings}

Mixture modeling suggested that modeled precision rather than guessing was responsible for the difference in memory quality between the two states. As the validity of mixture modeling has recently been questioned (Lawrence, 2010; Schurgin et al., 2018), we aimed to assess the trajectory of response precision across reports by accounting for differences in subjective confidence ratings. Specifically, we tested the trajectory for response precision across reports when subjects were confident that they did remember the item, i.e., when according to their self-report they did not guess. We evaluated response precision across successive reports separately for each level of confidence rating and set size. When participants gave a rating of "1", "2" or "3", indicating low, medium or high confidence, respectively, we found that response precision decreased significantly across reports in both set size conditions, except for low confidence in the set size two condition (confidence rating of "1": main effect of report position for set size two: $F(1,9)=5.1, p=$ $.0512, \eta_{p}^{2}=.36$, and for set size four: $F(1.4,12.4)=3.0, p=.097, \eta_{p}^{2}=.25$; confidence rating of "2" 


\section{Figure 4}

a

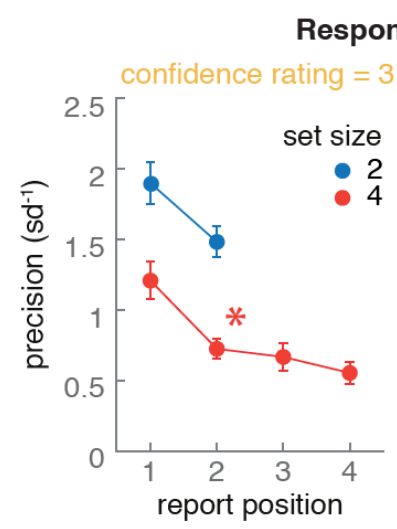

Response precision

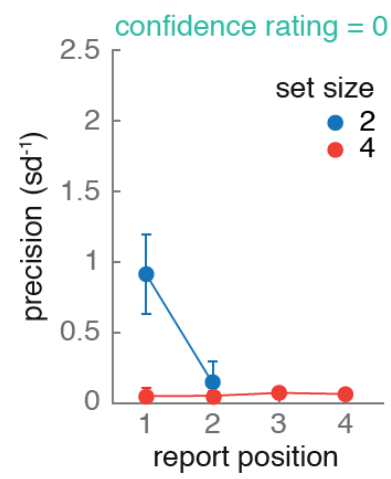

b

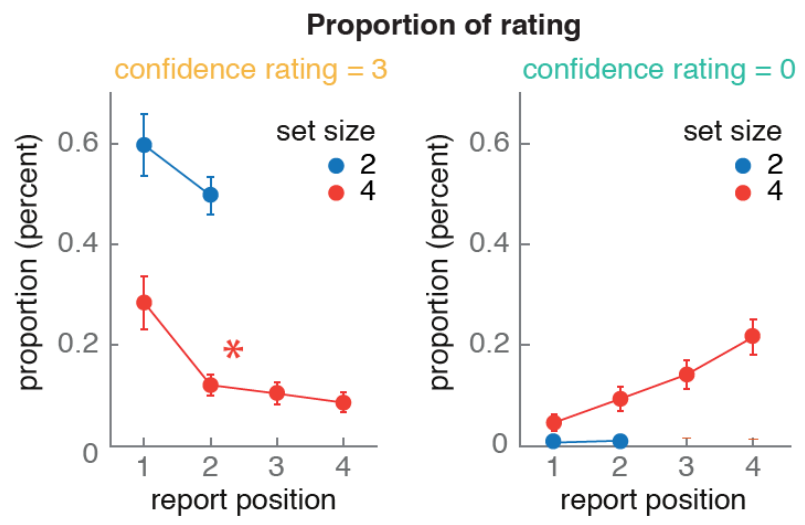

Figure 4. Response precision for the two extreme ratings and their proportions. a) Response precision that was accompanied with a confidence rating of 3 (left) and a confidence rating of 0 (right), indicating highest memory quality and forgetting, respectively. b) Proportion of confidence rating of 3 (left) and confidence rating of 0 (right). Error bars indicate standard error of the mean across participants. Asterisk denotes an advantage of a discontinuous over a continuous model for the decline trajectory across reports.

main effect of report position for set size two: $F(1,9)=49.9, p=.0001, \eta_{p}^{2}=.85$, and set size four: $\left.F(1.6,14.1)=14.2, p<.0001, \eta_{p}^{2}=.61\right)$; confidence rating of "3": main effect of report position for set size two: $F(1,9)=22.6, p=.0010, \eta_{p}^{2}=.72$, and for set size four: $F(3,27)=20.9, p<.0001$, $\left.\eta_{p}^{2}=.70\right)$. In contrast, we did not find such a decrease for the confidence rating of "0", corresponding to forgetting (see Fig. 4b, main effect of report position for set size four: $F(3,21)=0.12, p=$ $.948, \eta_{p}^{2}=.02$; note: because participants reported a rating of "0" very rarely in the set size two condition, we could not compute the corresponding ANOVA). Again and most importantly, for the set size four condition, we observed a steep drop of response precision from the first to the second report and a slight decrease thereafter only if participants reported to have low, medium or high confidence about the memorized orientation but not if they reported to have forgotten the orientation. These observations were supported by the model comparisons that favored the discontinuous over the continuous model for confidence ratings of "1", "2" and "3" (confidence rating of " 1 ": $\triangle \mathrm{AIC}=-10.09, \mathrm{X}^{2}(2)=14.09, p=.0009, \mathrm{c}=0.071$; confidence rating of "2": $\triangle \mathrm{AIC}=-6.38, \mathrm{X}^{2}(2)=$ $10.38, p=.006, \mathrm{C}=0.226$; confidence rating of "3": $\triangle \mathrm{AIC}=-2.44, \mathrm{X}^{2}(2)=6.44, p=.040, \mathrm{C}=0.463$ ). In contrast, comparisons favored the continuous over the discontinuous model for the confidence rating of "0" $\left(\triangle \mathrm{AIC}=4.00, \mathrm{X}^{2}(2)=0.00, p=1.000, \mathrm{c}=0.000\right)$.

\section{Reported forgetting increases continuously}

Mixture modeling suggested that only guessing increased continuously across reports. To exclude the possibility that this resulted from inaccurate estimation of the guessing parameters (Lawrence, 2010), we assessed the proportion with which participants rated an item as forgotten (confidence rating $=0$ ). The proportion of subjective forgetting did not increase significantly across reports for set size 2 (main effect of report precision: $F(1,9)=5.9, p=0.04, \eta_{p}^{2}=.40$ ) but did increase significantly for set size 4 (main effect for report precision: $F(1.4,12.4)=17.9, p<.001$, $\eta_{p}^{2}=.67$ ) (Fig. 4a, right panel). Hence, each report increased the probability of forgetting an item. Importantly, this increase of subjective forgetting in set size 4 was best captured by a continuous as compared to a discontinuous model $\left(\Delta \mathrm{AIC}=3.3, \mathrm{X}^{2}(2)=0.7, p=.73, \mathrm{c}=0\right)$. In line with the 
mixture modeling results, it demonstrates that the increase of items reported as forgotten does not reflect a difference between the states in working memory. In contrast, the proportion of items that were rated with the highest confidence (confidence rating $=3$ ) significantly decreased for set size $2\left(F(1,9)=3.2, p=0.11, \eta_{p}^{2}=.26\right)$ and set size $4\left(F(1.1,9.5)=14.3, p=.004,, \eta_{p}^{2}=.61\right)$ across the report sequence (Fig. $4 \mathrm{a}$, left panel). Corroborating the findings of the average confidence ratings and the modeled precision form the mixture model, the decline in high confidence ratings was better captured by a discontinuous compared to a continuous model $(\triangle \mathrm{AIC}=-26.9$, $\left.\mathrm{X}^{2}(2)=30.9, p<.001, \mathrm{C}=0.15\right)$ indicating that states in working memory differ in their memory quality.

\section{Discussion}

Using subjective and objective measures incorporated into a whole-report procedure we showed that participants remembered items at two levels of memory quality depending on the report position. Specifically, the first remembered item was frequently reported with high response precision, and participants were highly confident about their memory quality. In contrast, subsequently reported items were reported with lower response precision and received lower confidence ratings. The steep drop in response precision from the first to the second report was also present when trials were excluded in which participants reported to have no memory. Similarly, mixture modeling revealed that in particular the modeled precision parameter that indicates the memory quality dropped steeply from the first to the second report but decreased only slightly thereafter. We observed this stereotypical trajectory consistently for different measures of memory quality across reports. It was better captured by a model assuming a discontinuous as compared to a continuous decrease, indicating that items were indeed retrieved from two qualitatively distinct states in working memory. In contrast, the proportion with which participants rated an item as forgotten and the guessing parameter from the mixture model changed continuously rather than discontinuously across reports. These results suggest that forgetting took place to the same extent with every report. Together, our results show that states in working memory diverge in memory quality rather than quantity.

Our findings of two states in working memory correspond well to general architecture models that distinguish between at least two states of working memory. Unsworth \& Engle (2007), for example, have suggested that one state in working memory, termed primary memory, serves to store few items by means of the continued allocation of attention. Items in primary memory can be directly accessed for processing. When attention is removed from primary memory (for example when a distracting task is performed), items are maintained in a second state, termed secondary memory. Importantly, to be processed, items from secondary memory have to be retrieved by a cue-dependent search process. This process is error-prone, leading to failed or incorrect retrieval. Cowan (1988) proposed a similar architecture model but used a different terminology, distinguishing between a focus of attention and activated long-term memory for the first and second state, respectively. According to both models, differences between states in working memory are due to sustained attention necessary for storage of items in the first state and due to errors occurring during item retrieval from the second state. However, architecture models so far have not specified whether attention or error-prone retrieval affects the memory quality or quantity of items stored in the different states. This is likely due to the fact that studies that have informed the 
architecture models lacked measures of memory quality. These studies have typically used discrete - mostly verbal - stimuli and recorded reaction times and accuracy rates as indices of attention removal and thus the need for retrieval to as well as its error-proneness. Here we used the advantage of the delayed-estimation technique, in which both the stimulus and the response space are continuous rather than discrete. We showed that items were remembered with a markedly different memory quality before versus after the first report (i.e., the distracting interference task), indicating that they were stored in qualitatively different states. This is in line with architecture models proposing that a removal of attention by an interference event transferred items from the first to the second state. Our novel contribution to these models is that the first state in working memory stores high-quality representations that are, however, highly susceptible to even a single interference event. In contrast, items transferred to the second state have a lower quality but are more robust even against multiple interference events. These events, however, continuously increased the probability of forgetting, i.e., every report increased the subjective and objective measures of guessing to the same extent. This observation is also in line with architecture models proposing that retrieving items from the second state is an error-prone process that may interfere with all concurrently held items in the second state, hence, the chance of forgetting accumulates continuously with every report. According to architecture models one could also expect that every report will disrupt the binding between the item's content (i.e., orientation) and its cued feature (i.e., spatial position), making participants prone to retrieve the wrong item. However, we observed that the misreport parameter from mixture modeling was mostly unaffected by report position.

Given the importance of interference for the distinction between high versus low memory quality storage, we sought to characterize in detail the interference processes that are responsible for the transfer of items from the first to the second state in working memory. In our previous study (Peters et al., 2018) we showed in two experiments that the steep drop in response precision from the first to the second report was due to the executive interference. Specifically, we have implemented executive interference by asking participants to orient a Gabor probe vertically (Experiment 3) or to change the color of a patch to blue (Experiment 4) during the retention period before first report. We found that executive interference successfully mimicked the processes involved in the first report, as after executive interference the discontinuity in response precision between the first and subsequent reports vanished. In terms of the two-state interpretation, all items in working memory could only be reported from the second state. This was in contrast to other interference events, like passive viewing of a moving Gabor probe (perceptual interference) or a prolonged retention interval (longer delay). Under both conditions, participants were still able to report items from the first state, and the discontinuity between the first and second item was still observed. Consequently, the steep drop from the first to the second report that we observed in memory quality but not in guessing rate in the present study can be attributed to executive processes during the first report. Architecture models suggest that the successful retention of items in the first state requires sustained attention. Thus, whenever an executive interference task interrupts sustained attention, e.g. by redirecting attentional resources to novel input, memory items lose their high resolution and fall into the second state of lower resolution. An interesting question for future research would be to specify the nature of the executive attention mechanisms that are responsible for transferring items from the first to the second state. 
Another interesting issue regards the question whether different states refer to different levels of activation of a single item as proposed by the architecture models or to multiple representations of an item held separately in different stores. Consequently, does the transfer from the first to the second state indicate a loss of activation resulting in a weaker memory quality? Or alternatively, does it indicate that items are held concurrently in a high and a low-quality store but after interference the access to the high-quality representation is eliminated? Recent studies on the neuronal correlates of working memory have shown that task-relevant contents are maintained in multiple brain regions including primary sensory and parietal and frontal cortices (for review see Christophel, Klink, Spitzer, Roelfsema, \& Haynes, 2017). However, they also indicate that item representations are not simply duplicated across several brain regions but rather that representations differ in their level of abstraction with sensory regions encoding low-level sensory features and frontal and parietal regions encoding a more abstract and categorical format. Support for this view came from Bae \& Luck (2018) who showed that visual interference did not only reduce the memory quality but also increased the bias towards cardinal orientations suggesting an access to more categorial representations. Future research might test whether and why removal of attention impairs low-level sensory representations in sensory cortex but leaves abstract representations in frontal and parietal cortex unchanged.

For many cognitive tasks subjects typically memorize several task-relevant items. We showed that memory quality of these items varies systematically with the order with which the items are accessed and used. The sequential whole-report procedure thus provides a convenient way to measure the capacity of the two states in visual working memory. This approach also allows calculating an individual slope of decline in memory quality across reports. Future research might assess whether such an individual slope parameter is a promising measure to explore the underlying sources of working memory capacity limitation. However, so far only few studies have employed sequential reports from visual working memory (e.g., Adam, Mance, Fukuda, \& Vogel, 2015; Fougnie, Suchow, \& Alvarez, 2012; Woodman \& Vecera, 2011) with only one study, to the best of our knowledge, that also systematically investigated memory precision as a function of report order (Adam et al., 2017). In line with our findings, they found that precision dropped steeply from the first to the second report and declined slightly thereafter. This finding was obtained only when the item report order was randomized (Experiment 2 of Adam et al., 2017). In contrast, when participants could determine the report order (Experiment 1 of Adam et al., 2017), precision declined less steeply from the first to the second report and was remarkably comparable between set sizes. This suggests that the possibility to choose the report order might lead to the use of recall and encoding strategies that influence the trajectory of precision in addition to reports from different states.

The present study provides novel evidence that states in working memory differ specifically with respect to their levels of memory quality. While removal of attention by report/interference determines whether an item is held in the first state with high quality or in the second state with lower quality, further retrieval from the second state has little impact on memory quality but leads to forgetting. 


\section{References}

Adam, K. C. S., Vogel, E. K., \& Awh, E. (2017). Clear evidence for item limits in visual working memory. Cogn Psychol, 97, 79-97. http://doi.org/10.1016/j.cogpsych.2017.07.001

Adam, K. C., Mance, I., Fukuda, K., \& Vogel, E. K. (2015). The Contribution of Attentional Lapses to Individual Differences in Visual Working Memory Capacity. $J$ Cogn Neurosci, 1-16. http://doi.org/10.1162/jocn_a_00811

Bae, G.-Y., \& Luck, S. J. (2018). What happens to an individual visual working memory representation when it is interrupted? British Journal of Psychology, 7(2), 4785-20. http://doi.org/10.1111/bjop.12339

Bays, P. M., \& Husain, M. (2008). Dynamic shifts of limited working memory resources in human vision. Science, 321(5890), 851-854. http://doi.org/10.1126/science.1158023

Bays, P. M., Catalao, R. F. G., \& Husain, M. (2009). The precision of visual working memory is set by allocation of a shared resource. J Vis, 9(10), 7-7. http://doi.org/10.1167/9.10.7

Bledowski, C., Rahm, B., \& Rowe, J. B. (2009). What "Works" in Working Memory? Separate Systems for Selection and Updating of Critical Information. Journal of Neuroscience, 29(43), 13735-13741. http://doi.org/10.1523/jneurosci.2547-09.2009

Christophel, T. B., Klink, P. C., Spitzer, B., Roelfsema, P. R., \& Haynes, J.-D. (2017). The Distributed Nature of Working Memory. Trends Cogn Sci, 1-14. http://doi.org/10.1016/j.tics.2016.12.007

Cowan, N. (1988). Evolving Conceptions of Memory Storage, Selective Attention, and Their Mutual Constraints within the Human Information-Processing System. Psychological Bulletin, 104(2), 163-191.

Fougnie, D., Suchow, J. W., \& Alvarez, G. A. (2012). Variability in the quality of visual working memory. Nat Commun, 3, 1229. http://doi.org/10.1038/ncomms2237

Lawrence, M. A. (2010). Estimating the probability and fidelity of memory. Behavior Research Methods, 42(4), 957-968. http://doi.org/10.3758/BRM.42.4.957

McElree, B. (2006). Accessing Recent Events, 46, 155-200. http://doi.org/10.1016/s00797421(06)46005-9

Nee, D. E., \& Jonides, J. (2014). Frontal-medial temporal interactions mediate transitions among representational states in short-term memory. J Neurosci, 34(23), 7964-7975. http://doi.org/10.1523/JNEUROSCI.0130-14.2014

Oberauer, K. (2002). Access to information in working memory: Exploring the focus of attention. J Exp Psychol Learn Mem Cogn, 28(3), 411-421. http://doi.org/10.1037//0278-7393.28.3.411

Peters, B., Rahm, B., Czoschke, S., Barnes, C., Kaiser, J., \& Bledowski, C. (2018). Sequential Whole Report Accesses Different States in Visual Working Memory. J Exp Psychol Learn Mem Cogn, 44(4), 588, http://doi.org/10.1037/xlm0000466

Rademaker, R. L., Tredway, C. H., \& Tong, F. (2012). Introspective judgments predict the precision and likelihood of successful maintenance of visual working memory. $J$ Vis, 12(13), 21. http://doi.org/10.1167/12.13.21

Schurgin, M. W., Wixted, J. T., \& Brady, T. F. (2018). Psychological Scaling Reveals a Single Parameter Framework For Visual Working Memory. bioRxiv, 1-25. http://doi.org/10.1101/325472 
Unsworth, N., \& Engle, R. W. (2007). The nature of individual differences in working memory capacity: Active maintenance in primary memory and controlled search from secondary memory. Psychol Rev, 114(1), 104-132. http://doi.org/10.1037/0033-295X.114.1.104

van den Berg, R., van den Berg, \& Ma, W. J. (2017). Fechner's Law in Metacognition: A Quantitative Model of Visual Working Memory Confidence. Psychol Rev, 1-18. http://doi.org/10.1037/rev0000060.supp

Wilken, P., \& Ma, W. J. (2004). A detection theory account of change detection. J Vis, 4(12), $1120-1135$.

Woodman, G. F., \& Vecera, S. P. (2011). The cost of accessing an object's feature stored in visual working memory. Visual Cognition, 19(1), 1-12. http://doi.org/10.1080/13506285.2010.521140

Zhang, W., \& Luck, S. J. (2008). Discrete fixed-resolution representations in visual working memory. Nature, 453(7192), 233-235. http://doi.org/10.1038/nature06860 